\title{
Ex vivo experimental study on the Thulium laser system: new horizons for interventional endoscopy (with videos)
}

\section{(ㄷ)(1) $\odot$}

Authors

Gian Eugenio Tontini ${ }^{1}$, Helmut Neumann ${ }^{2}$, Alessandro Rimondi ${ }^{1,3}$, Sara Vavassori ${ }^{1,3}$, Barbara Bruni ${ }^{4}$, Gregorio Cattignoli ${ }^{5}$, Ping-Hong Zhou ${ }^{6}$, Luca Pastorelli ${ }^{1,7}$, Maurizio Vecchi ${ }^{1,7}$

Institutions

1 Gastroenterology \& Digestive Endoscopy Unit, IRCCS Policlinico San Donato, San Donato Milanese, Milano, Italy

2 Interventional Endoscopy Center, University Hospital Mainz, Mainz, Germany

3 University of Milan

4 Pathology and Citodiagnostic Unit, IRCCS Policlinico San Donato, San Donato Milanese, Milano, Italy

5 Surgical Division, Quanta System S.p.A., Varese, Italy

6 Endoscopy Center, Zhongshan Hospital, Fudan University, Shanghai, China

7 Department of Biomedical Sciences for the Health, University of Milan, Italy

submitted 11.1.2017

accepted after revision 20.3.2017

\section{Bibliography}

DOI https://doi.org/10.1055/s-0043-106738 |

Endoscopy International Open 2017; 05: E410-E415

(c) Georg Thieme Verlag KG Stuttgart · New York

ISSN 2364-3722

Corresponding author

Gian Eugenio Tontini, MD, PhD, Gastroenterology and Digestive Endoscopy Unit, IRCCS Policlinico San Donato, Via Morandi 30, 20097 San Donato Milanese, Milano, Italy Fax: +39-02-52774655

gianeugeniotontini@gmail.com

\section{ABSTRACT}

Background and study aims The Thulium laser system (TLS) is an emerging interventional tool adopted in many surgical specialties. Its $2.0-\mu \mathrm{m}$ wavelength allows precise coagulation (0.2-0.4 mm in depth) and cutting, limiting the possibilities of collateral injuries. We tested the impact of the TLS for gastric endoscopic submucosal dissection (ESD) and per oral endoscopic myotomy (POEM) ex vivo in pigs.

Materials and methods Ex vivo porcine stomach and esophagus models underwent 2 POEMs, and 3 ESDs (mean diameter $3.5 \mathrm{~cm}$ ) with TLS using a $272-\mu \mathrm{m}$ and a $365-\mu \mathrm{m}$ thick optical fibers. Both continuous and pulsed laser emission were evaluated. Subsequent histopathological analysis was performed by an expert GI pathologist on the whole porcine models.

Results Complete POEMs and gastric ESDs were successfully performed in all cases in 30 to 70 and 15 to 20 minutes. Both optical fibers were equally effective and precise. The best power output for mucosal incision was 25 to $30 \mathrm{~W}$ during ESD and $25 \mathrm{~W}$ for POEM using continuous laser emission. During submucosal dissection and tunneling the favorite power output was $20 \mathrm{~W}$ and 15 to $20 \mathrm{~W}$, respectively, operating in continuous mode. No transmural perforation occurred throughout the operations and histopathology confirmed the absence of accidental muscular layer damage.

Conclusions The TLS stands out as a precise and manageable instrument in ex vivo models. This technique appears to be a promising tool for advanced interventional endoscopy.

\section{Introduction}

Per oral endoscopic myotomy (POEM) and endoscopic submucosal dissection (ESD) are well-established techniques in Gl endoscopy [1-3]. These procedures are possible by means of electrosurgical devices able to transform high frequency alternate electrical current into thermal energy for either ablation, coagulation or cutting purposes [4]. At the current state of the art, thermal energy is conveyed within the tissue by direct con- tact of conductive electrosurgical probes in both POEM and ESD $[1,2]$. In standard endoscopic settings, electrosurgical accessories are safely employed in many clinical applications, also including hemostasis, tissue ablation and tumor debulking, with rare complications. However, the thermal energy delivered by conductive probes within the tissue may provoke lateral and transmural injuries ( $\geq 2-3 \mathrm{~mm}$ ) and eventually bleeding and perforation. Such complications depend on many factors including duration of application, power settings, rate and mag- 


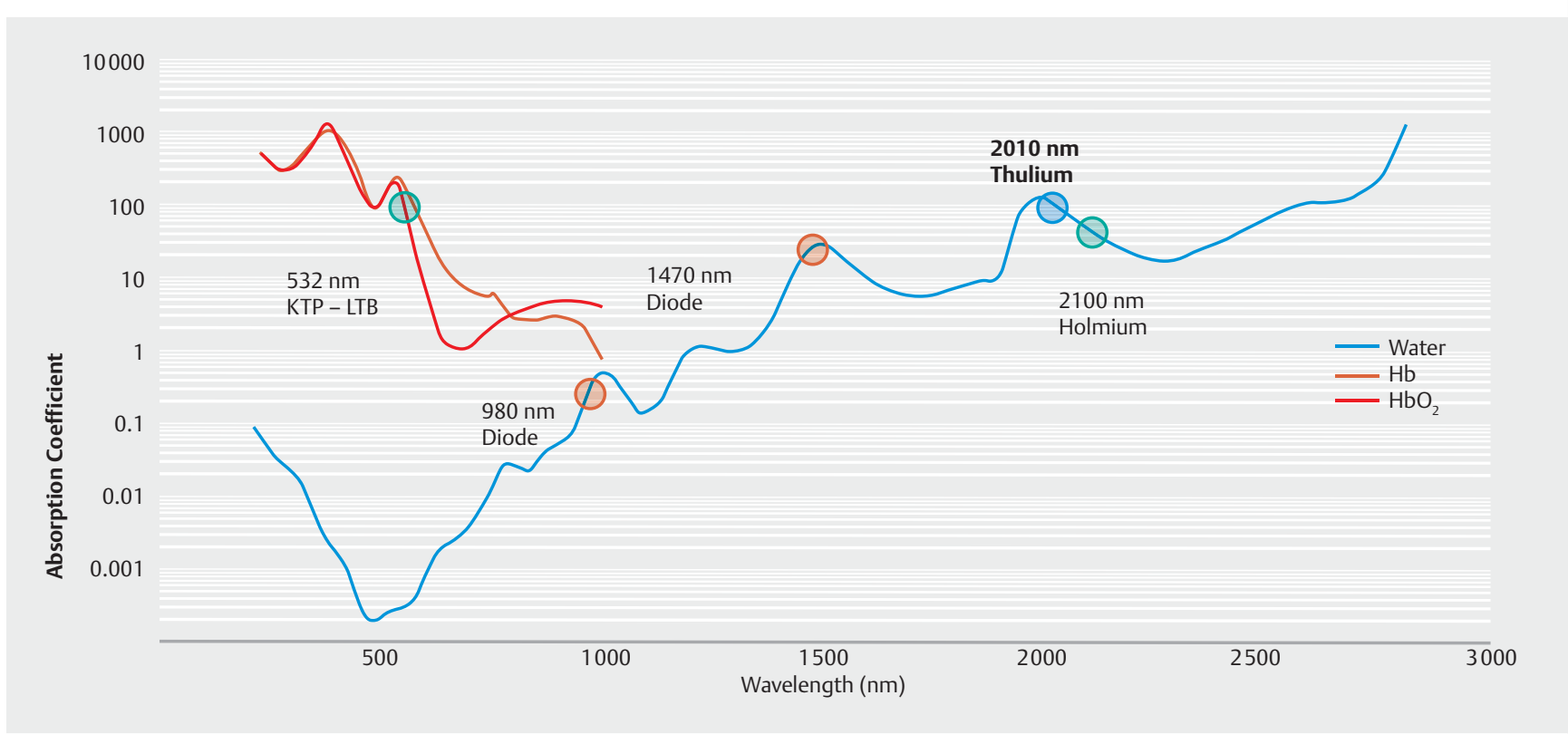

- Fig. 1 The role of electromagnetic wavelength on laser absorption coefficient for hemoglobin and water.

nitude of electrosurgical current, tissue resistance and probe properties. Accordingly, prolonged administration of thermal energy onto the same area is not generally advisable [4]. Nonetheless, in challenging endoscopic tasks, such as ESD and POEM, the extensive and sustained use of electrosurgical devices may account for a certain burden of complications [4].

The Thulium-doped yttrium-aluminium-garnet laser system (TLS), a recently developed therapeutic laser, earns a chance to overcome these limitations. The TLS acts with a continuous or pulsed wave mode at $2.0-\mu \mathrm{m}$ ( $\triangleright$ Fig. 1) [5]. Already employed in surgery and endourology, this new laser technique has gained rapid consensus among specialists as a reliable therapeutic tool for surgical resection, vaporesection, ablation, and coagulation, thus substituting less precise laser techniques (e. g., Holmium laser) [6 - 11]. To our knowledge, the experience on the use of the TLS in Gl endoscopy is very limited $[12,13]$.

In this study, we evaluated for the first time the feasibility of TLS-assisted gastric ESD and POEM in standard experimental models assessing the laser-induced tissue damage to the submucosal and muscle layer.

\section{Materials and methods}

All procedures were conducted ex vivo in an established experimental setting by using two porcine models of the upper $\mathrm{Gl}$ tract. The TLS applied during this study was the Cyber TM ${ }^{\circledR}$ (Quanta System SpA, Varese, Italy) ( $\bullet$ Table 1 ). This laser system acts with a $2.0-\mu \mathrm{m}$ wavelength providing a wide range of power settings $(1-200 \mathrm{~W})$ suitable for both surgical and endoscopic uses [8-13]. For the purposes of the present study, we evaluated power settings ranging from 5 to $30 \mathrm{~W}$ emitted with either a continued or a pulsed laser configuration. Various flexible optical fibers (272 and $365 \mu \mathrm{m}$-thick) were also tested.
- Table 1 Specifications of the Cyber TM thulium laser system.

\begin{tabular}{|l|l|}
\hline \multicolumn{2}{|l|}{ Cyber TM-Thulium Laser System } \\
\hline Wavelength & $2010 \mathrm{~nm}$-Thulium \\
\hline Power output & Up to $200 \mathrm{~W}$ (1 to $5 \mathrm{~W}$ increment steps) \\
\hline Treatment mode & Single wavelength \\
\hline Treatment mode & Continuous or pulsed wave \\
\hline Aiming beam & Red $(650 \mathrm{~nm})$ or green $(532 \mathrm{~nm})$ \\
\hline Optical fibers & $\begin{array}{l}\text { Frontal emission: } \varnothing 200 \text { to } 1000 \mu \mathrm{m} \text { (reusable) } \\
\text { Lateral emission: } \varnothing 600 \mu \mathrm{m}(\text { disposable) }\end{array}$ \\
\hline $\begin{array}{l}\text { Dimensions } \\
\text { Weight }\end{array}$ & $55 \times 75 \times 110 \mathrm{~cm} / 200 \mathrm{~kg}$ \\
\hline
\end{tabular}

Two Western endoscopists were involved, 1 with substantial skills in either gastric ESD ( $40-50$ procedures) or POEM (20 - 30 procedures), and 1 with no expertise with these techniques. Both operators had very limited previous experience on the ex vivo use of this new endoscopic device. All procedures were conducted using standard high-definition gastroscopes and digitally recorded.

The ESD of 3 large (mean $3.5 \mathrm{~cm}$, range $3.3-3.6 \mathrm{~cm}$ ) injection-induced (saline solution) lesions of the stomach was performed following a standard technique in two ex vivo models. The TLS-guided POEM was simulated performing an esophageal incision and submucosal tunnelling down to the gastroesophageal junction in 2 ex vivo models.

Following the endoscopic procedures, an expert gastrointestinal pathologist performed the histopathological analysis on the entire stomachs looking for signs of transmural perfora- 
- Table 2 ESD and POEMs specifications.

\begin{tabular}{|l|c|c|c|c|}
\hline & Operator & Dimension & Time & Outcomes \\
\hline ESD No. 1 & 1 & $3.6 \mathrm{~cm}$ & 50 minutes & $\begin{array}{l}\text { No transmural perforation; No tissue damage in muscolaris propria } \\
\text { and submucosa. }\end{array}$ \\
\hline ESD No. 2 & 1 & $3.3 \mathrm{~cm}$ & 30 minutes & \\
\hline ESD No. 3 & 2 & $3.5 \mathrm{~cm}$ & 70 minutes & \multirow{2}{*}{ No perforation in outward and luminal layers. } \\
\hline POEM No. 1 & 1 & - & 20 minutes & \\
\hline POEM No. 2 & 2 & - & 30 minutes & \\
\hline $\begin{array}{l}\text { Operator No.1 - Expert gastrointestinal endoscopist with initial expertise in ESD and POEM. } \\
\text { Operator No.2 - Expert gastrointestinal endoscopist with no previous experience in ESD and POEM }\end{array}$
\end{tabular}

tion or tissue injuries affecting the muscolaris propria or the deepest submucosal layer.

\section{Results}

Complete en bloc gastric ESD was feasible in all cases. Both endoscopists reported an easy maneuverability regardless of their expertise in ESD procedures ( $\vee$ Video 1$)$. ESD operative time ranged between 30 and 70 minutes according to the operator's expertise in ESD as well as in TLS-assisted ESD ( $\bullet$ Table 2). More in detail, 10 to 30 minutes were needed for gastric mucosal incision, and 20 to 40 minutes for gastric lesions dissection.

The lesions' border was traced using a 5-W laser output. The $272-\mu \mathrm{m}$ and the $365-\mu \mathrm{m}$ thick optical fibers appeared equally effective for both mucosal incision and submucosal dissection using various power settings $(20-35 \mathrm{~W}$ for incision, $15-25 \mathrm{~W}$ for dissection). A progressive increase in the favorite power setting was observed for both operators over the course of their performance: the best-perceived power output turned from $20 \mathrm{~W}$ to 25 to $30 \mathrm{~W}$ for mucosal incision and from 15 to $20 \mathrm{~W}$ for submucosal dissection. Laser emission in continuous mode resulted in clearer cutting lines and was adopted by both operators instead of the pulse modality.

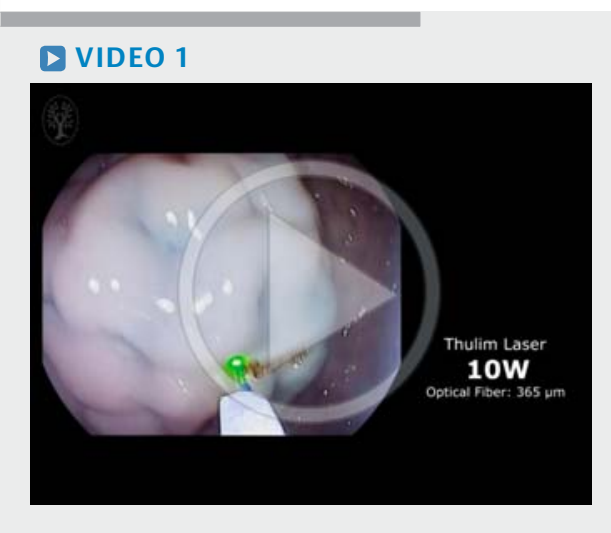

- Video 1: Thulium-assisted endoscopic submucosal dissection in ex vivo porcine model.

Online content viewable at: https://www.thieme-connect.com/ DOI/DOI?10.1055/s-0043-106738
Excessive smoke, albeit never obscuring the endoscopic view, was observed during mucosal incisions when laser emission was used in air on dry tissue ( $\bullet$ Fig.2). On the contrary, smoke emission was largely less when submucosal dissection was done. Histopathology performed on the gastric wall corresponding to the 3e ESD treatments confirmed the absence of transmural perforation, thereby showing no tissue damage within the muscolaris propria and the deepest submucosal layer ( $\vee$ Fig. 3).

Both esophageal incisions and submucosal tunneling down to the gastroesophageal junction were also feasible within 20 to 30 minutes and regardless of the operators' expertise ( $\vee$ Video 2, > Fig.4). Even in this setting, the 272- $\mu \mathrm{m}$ and 365$\mu \mathrm{m}$ thick optical fibers appeared equally effective for both mucosal incision and submucosal tunneling using various power settings (20-35 W for incision, $15-25 \mathrm{~W}$ for dissection). The best-perceived power setting was $25 \mathrm{~W}$ for mucosal incision and 15-20W for submucosal tunneling, operating in continuous mode. As for gastric ESD, smoke emission was evident yet non-influential during mucosal incision and negligible during submucosal tunneling.

As confirmed by histopathology, no transmural perforation, neither to the luminal side (i.e., mucosal) occurred ( $\bullet$ Table 2 , > Fig. 5).

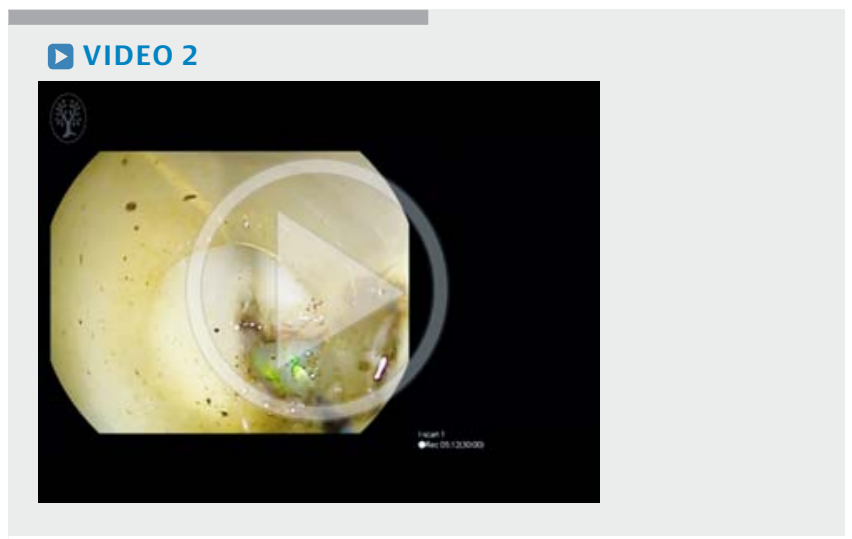

Video 2: Thulium-assisted per oral endoscopic myotomy in ex vivo porcine model.

Online content viewable at: https://www.thieme-connect.com/ DOI/DOI?10.1055/s-0043-106738 

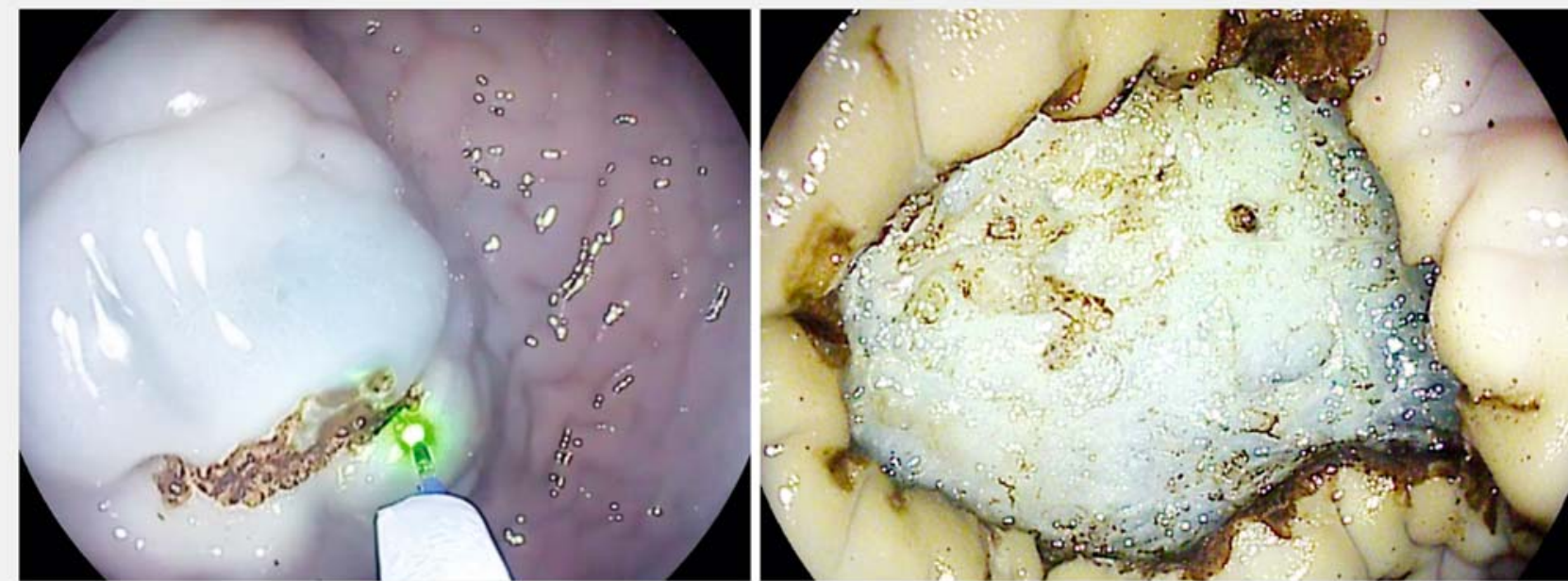

Fig. 2 Representative still image from Video 1 - Thulium-assisted endoscopic submucosal dissection: incision (left side) and final result (right side).

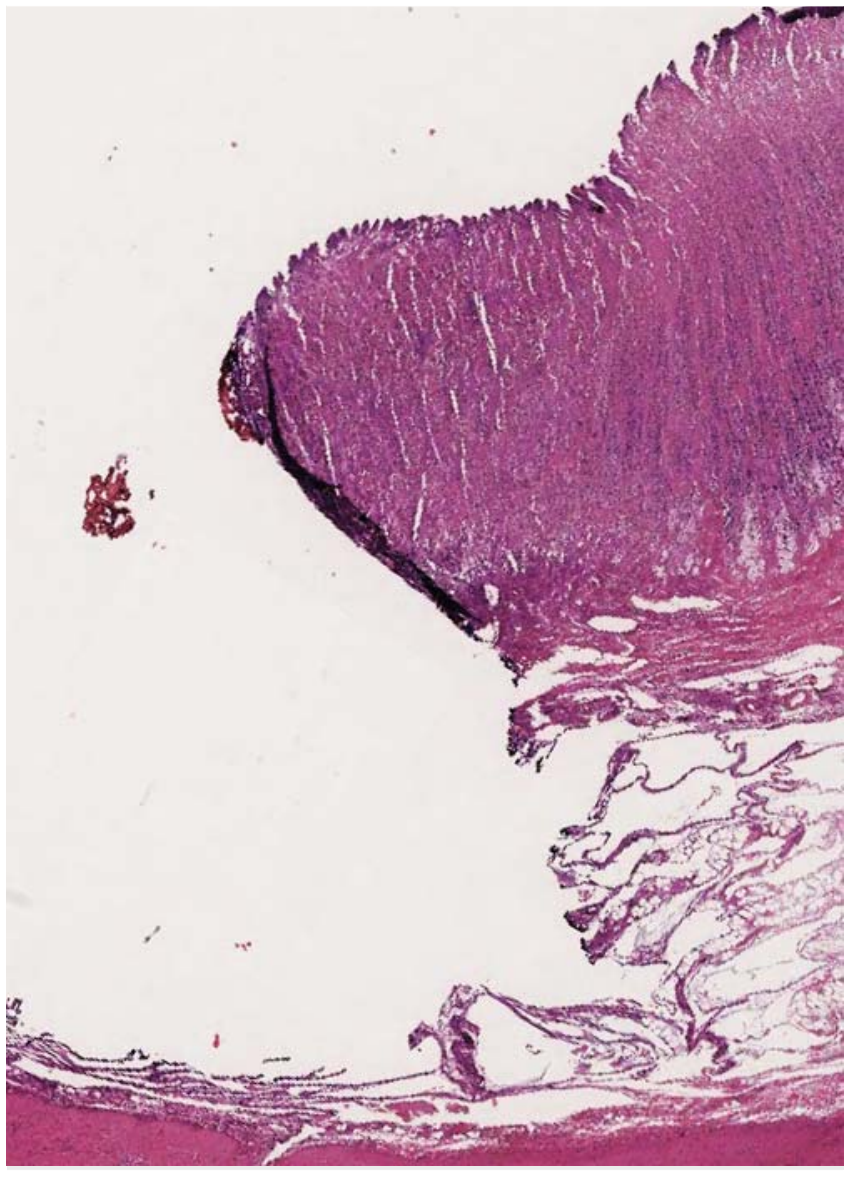

- Fig. 3 Peripheral section of the porcine gastric wall following the Thulium-assisted ESD procedure showing no tissue injury affecting the deepest submucosal layer and muscolaris propria (Hematoxylin \& Eosin, $5 \mathrm{X})$.

\section{Discussion}

Previous surgical and endourological in vivo studies demonstrated how laser technology may be considered an effective and successful alternative to standard electrosurgical devices [5-8]. Recently, we evaluated the potential of the TLS in GI endoscopy. The TLS provided favorable performance in ex vivo models as compared to argon plasma coagulation and was successfully adopted for the endoscopic treatment of non-variceal upper GI bleeding in vivo [13]. In particular, the $2.0-\mu \mathrm{m}$ wavelength of the TLS allows a very confined vaporesective and hemostatic effect on the targeted mucosal surface, reducing the chance of unexpected injuries on the underlying tissue [13]. Another pilot study conducted in vivo showed that the Thulium laser is a feasible tool for ESD in early gastric epithelial neoplasia. In that paper from Korea, curative resection was achieved in 9/10 patients without relevant complications. All procedures were performed by a single very experienced endoscopist (>1000 ESD); nonetheless, authors reported a prolonged incision time to avoid accidental deep tissue injuries [12]. We therefore conducted a feasibility study in ex vivo models to evaluate the effect of TLS-assisted ESD on deeper gastric layers and to test its performance even in less expert hands ( $<50$ ESD).

This study confirms both the feasibility and safety of TLS in performing ESD and POEM. Either mucosal incision and submucosal dissection or submucosal tunneling were easily performed regardless of the operator's expertise. The use of this non-contact device for mucosal incision and submucosal dissection allowed the endoscopists to easily assess the conditions of the digestive wall in real time, while operating throughout all the procedure, thereby reducing the risk of unnoticed deep tissue injuries. Consistently, histopathological analysis on the entire upper gastrointestinalmodels revealed that TLS has no impact on the deeper layers during ESD and POEM.

These initial results support future implementations of laserassisted techniques for advanced endoscopic treatments. In 


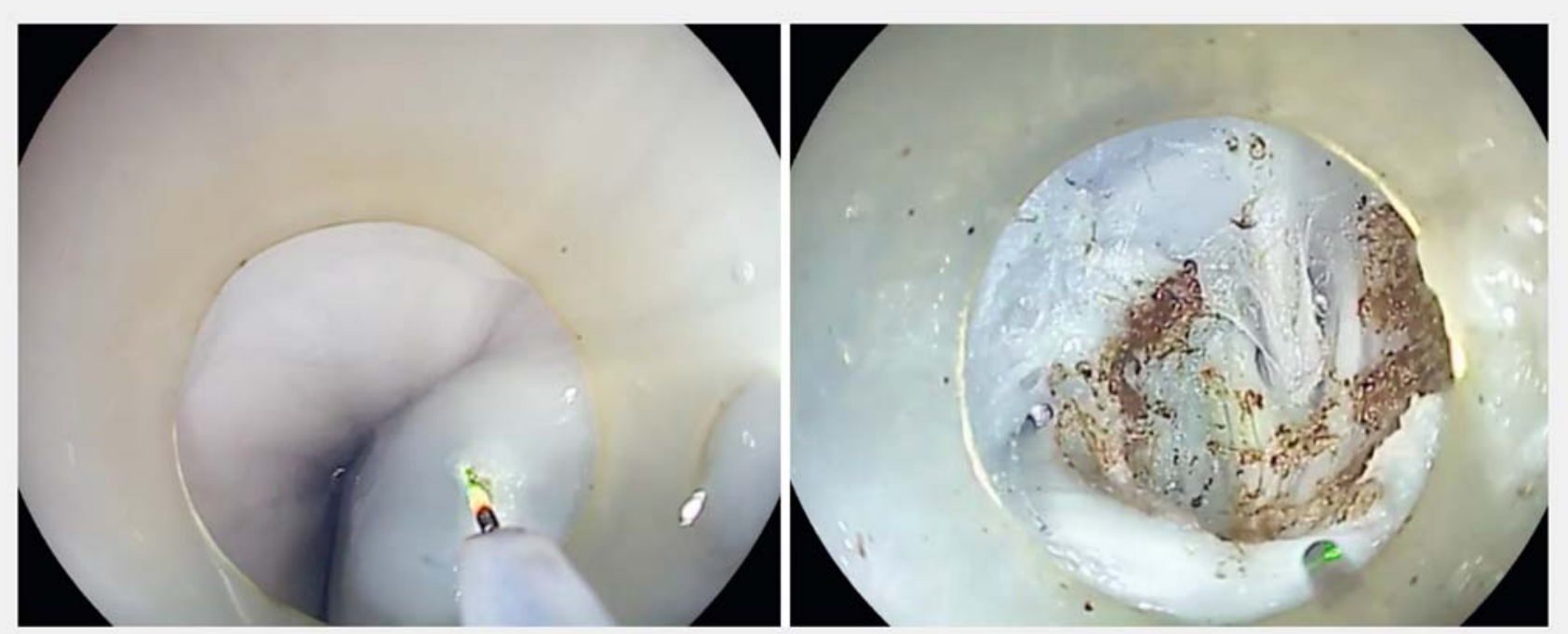

- Fig. 4 Representative still image from Video 2 - Thulium-assisted POEM: mucosal incision to expose the submucosal space $15 \mathrm{~cm}$ proximal to the gastroesophageal junction (left side) and submucosal tunneling up to the gastroesophageal junction (right side).

particular, the use of incision devices with very confined impact on deeper layers and high hemostatic efficacy can potentially improve both technical and clinical outcomes associated with ESD and POEM, especially when bleeding or severe fibrosis with muscle fibers adherent to the submucosal layer increase the risk of perforation $[14,15]$. Peculiar benefits of the TLS beyond additional laser systems include a continuous wave mode combined with the highest coefficient of absorption in water, meaning more precise cutting power and a higher hemostatic potential. On the contrary, the Holmium laser provides a pulsed wave mode at $2.1 \mu \mathrm{m}$, therefore inducing deeper coagulation on soft tissue ( $\triangleright$ Fig. 1 ) $[7,13]$.

Another matter associated with the use of laser systems for surgical purposes relies on the optical fibers durability. Optical fibers with limited duration show a progressive depletion of laser collimation, thereby spreading the laser beam on a less limited and symmetric target. On the contrary, we never changed the two probes ( 272 and $365 \mu \mathrm{m}$ thick) during this study. Experiences from laser enucleation of the prostate showed that the TLS optical fibers maintain similar performance during prolonged uses (up to 60 minutes under continuous emission in water immersion) with very high-power settings (>100 W) [9]. In addition, the TLS reusable optical fibers recover their original performance just cleaving the terminal $1 \mathrm{~cm}$ of the tip (using a dedicated ceramic blade) following several and prolonged uses. All reusable TLS optical fibers can be sterilized (e. g., steam and gas-plasma sterilization). To date, there is no study assessing the cumulative use (in term of operative time or power output) associated with a significant reduction of the TLS optical fiber performances. Consistently, there is no standardized limit beyond which a revision of the optical laser tip is recommended. Based on our experience, additional variables might also interfere with this correlation, such as their use in air versus liquid, probe overheating and previous contact of the optical fiber tip with organic tissues.
Remarkably, smoke emission and evacuation could represent an issue for prolonged TLS use in air, especially on dry tissue and during mucosal incision either for in vivo [12] or ex vivo settings [13]. In our experience, both water irrigation through the ancillary working channel of the endoscope and lasing in water immersion were smoke-free procedures. In fact, no accessory device assisting the use of frontal optical fibers during endoscopic laser treatments has been planned so far (e.g., for irrigation, injection, smoke evacuation, rotation, orientation). To date, needle-knife or paintbrush like laser emissions can be delivered only straight on the optical fiber placed at the tip of the endoscope's working channel.

These aspects together with the very limited operators' experience with use of this novel endoscopic tool may have played a role in determining the wide range of time to achieve a complete gastric ESD within this ex vivo study. The use of bloodless experimental settings usually results in shorter operative time. On the contrary, the observed ESD operative time of 30 to 70 minutes is in line with those observed for in vivo TLSassisted ESD of 1 to $2 \mathrm{~cm}$ gastric epithelial neoplasia reported by Cho J-H et al. (median 49 minutes, range 35-2013 minutes) [12]. However, the second TLS-assisted ESD performed by the same operator was much quicker than the previous one in our study, thereby suggesting a substantial learning curve effect related to the use of this novel endoscopic tool for ESD.

Notably, the TLS wavelength of $2 \mu \mathrm{m}$ has an innate hemostatic effect and has already shown promising performance when adopted to treat ongoing bleedings in vivo [5-13]. Accordingly, the main limitation of this feasibility study relies on its bloodless experimental setting, which could have been not appropriate to highlight one of the potential key features of this technique, which is a more potent hemostatic effect. 


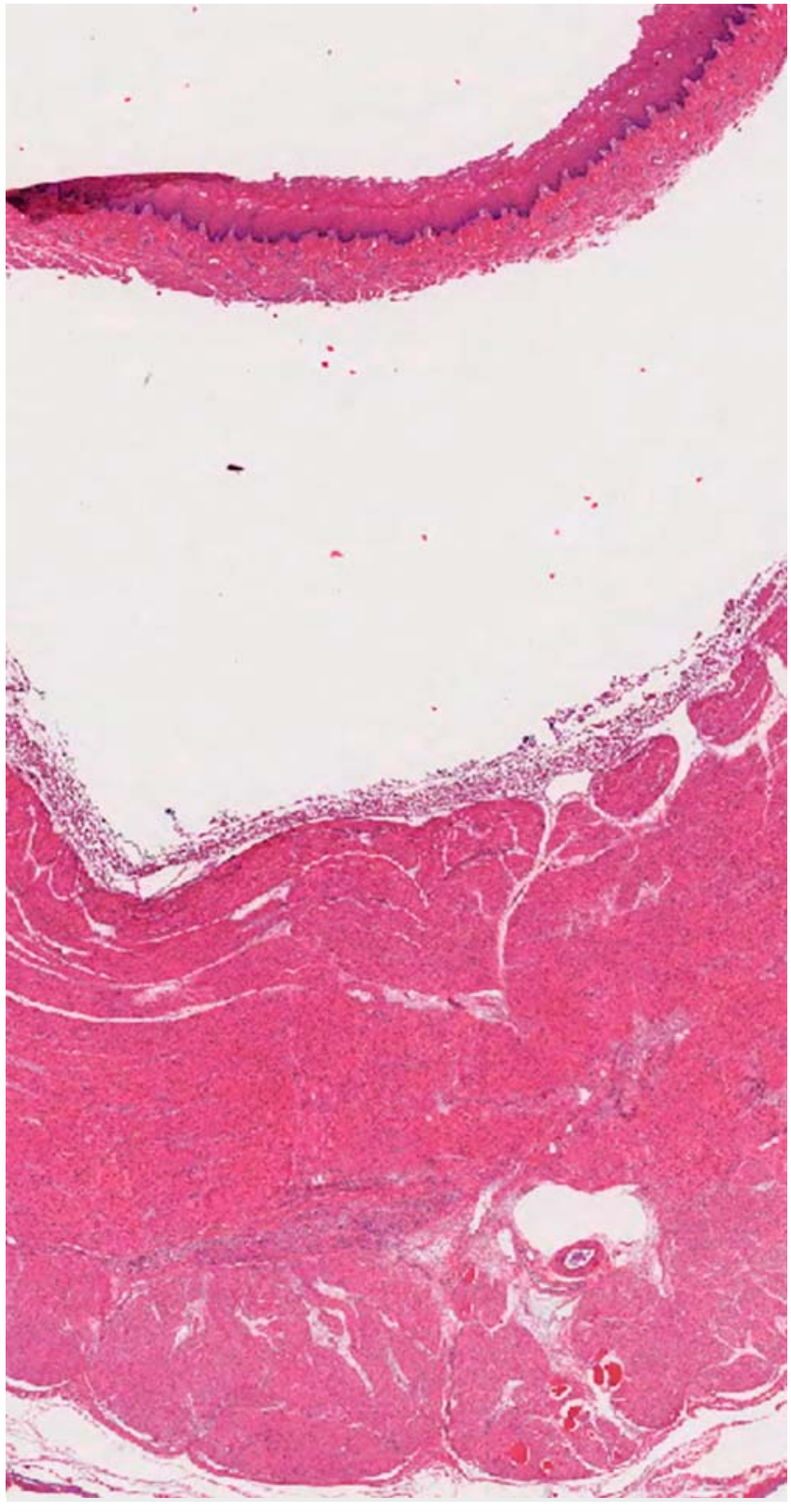

- Fig. 5 Peripheral section of the porcine esophageal wall following the Thulium-assisted POEM procedure showing no tissue injury (Hematoxylin \& Eosin, 5X).

\section{Conclusion}

In conclusion, our ex vivo experiences suggest that TLS is a precise and manageable endoscopic treatment device. By means of different optical fibers and power settings, the TLS appears perfectly suitable for several and heterogeneous endoscopic treatments, including ESD and POEM. Nonetheless, we advocate the need of in vivo preliminary assessment to confirm our ex vivo results. Should these results be replicated, large prospective trials will be required to assess the opportunity of adding TLS to endoscopists' toolbox.

\section{Acknowledgments}

This independent research has received no funding. Preliminary data from this work were presented at the following meetings: FISMAD 2016 (Naples, Italy - oral and poster presentations), UEGW 2016 (Vienna, Austria - poster presentation).

\section{Competing interests}

G.C. is currently employed at the Surgical Division of Quanta System. The remaining authors disclosed no financial relationships relevant to this publication.

\section{References}

[1] Chiu PWY, Inoue H, Rösch T. From POEM to POET: applications and perspectives for submucosal tunnel endoscopy. Endoscopy 2016; 48: $1134-1142$

[2] Maple JT, Abu Dayyeh BK, Chauhan SS. ASGE Technology Committee. et al. Endoscopic submucosal dissection, Gastrointest Endosc. 2015; 81: $1311-1325$

[3] Fujishiro M, Yasuda I. Congress report: Exchange of opinions in Tokyo, 2016 between Japan Gastroenterological Endoscopy Society (JGES) and counterparts from abroad. Dig Endosc 2016; 28: 642-644

[4] Tokar JL, Barth BA. ASGE Technology Committee. et al. Electrosurgical generators. Gastrointest Endosc 2013; 78: 197-208

[5] Fried NM, Murray KE. High-power thulium fiber laser ablation of urinary tissues at 1.94 microm. J Endourol 2005; 19: 25-31

[6] Xia S], Zhuo J, Sun XW et al. Thulium laser versus standard transurethral resection of the prostate: a randomized prospective trial. Eur Urol 2008; 53: $382-389$

[7] Zhang F, Shao Q, Herrmann TR et al. Thulium laser versus holmium laser transurethral enucleation of the prostate: 18-month follow-up data of a single center. Urology 2012; 79: 869-874

[8] Muto G, Collura D, Giacobbe A et al. Thulium:yttrium-aluminum-garnet laser for en bloc resection of bladder cancer: clinical and histopathologic advantages. Urology 2014; 83: $851-855$

[9] Carmignani L, Pastore AL, Picozzi SC et al. Thulium laser prostate enucleation in refractory urinary retention: Operative and functional outcomes in a large cohort of patients. Urology 2016; 93: 152-157

[10] Passacantilli E, Lapadula G, Caporlingua F et al. Preparation of nasoseptal flap in trans-sphenoidal surgery using $2-\mu$ thulium laser: technical note. Photomed Laser Surg 2015; 33: 220-223

[11] Kirschbaum A, Höchsmann N, Steinfeldt T et al. Investigations of initial airtightness after non-anatomic resection of lung parenchyma using a thulium-doped laser with different optical fibres. Lasers Med Sci 2016; 31: $1097-1103$

[12] Cho JH, Cho JY, Kim MY et al. Endoscopic submucosal dissection using a thulium laser: preliminary results of a new method for treatment of gastric epithelial neoplasia. Endoscopy 2013; 45: 725 - 728

[13] Tontini GE, Neumann H, Pastorelli L et al. Thulium laser in interventional endoscopy: animal and human studies. Endoscopy 2017; 49: $365-370$ [Epub ahead of print]

[14] Pimentel-Nunes P, Dinis-Ribeiro M, Ponchon T et al. Endoscopic submucosal dissection: European Society of Gastrointestinal Endoscopy (ESGE) Guideline. Endoscopy 2015; 47: 829-854

[15] Pannala R, Abu Dayyeh BK. ASGE Technology Committee. et al. Peroral endoscopic myotomy (with video). Gastrointest Endosc 2016; 83: $1051-1060$ 